\title{
Upper eyelid reconstruction using a combination of a nasal septal chondromucosal graft and a Fricke flap: a case report
}

Ju Ho Lee,

Sang Seok Woo,

Se Ho Shin,

Hyeon Jo Kim,

Jae Hyun Kim,

Seong Hwan Kim,

In Suck Suh

Department of Plastic and

Reconstructive Surgery, Kangnam

Sacred Heart Hospital, Hallym

University College of Medicine, Seoul, Korea
Sebaceous carcinoma is a malignant neoplasm that usually arises in the sebaceous glands of the eyelids. Its pathogenesis is unknown; however, irradiation history, immunosuppression, and use of diuretics are known risk factors. The mainstay of treatment for sebaceous carcinoma of the eyelid is wide surgical resection with a safety margin of 5 to $6 \mathrm{~mm}$, which often results in fullthickness defects. The reconstruction of a full-thickness defect of the eyelid should be approached using a three-lamella method: a mucosal component replacing the conjunctiva, a cartilage component for the tarsal plate, and a flap or skin graft for the skin of the eyelid. In this case, a fullthickness defect of the upper eyelid was reconstructed after tumor removal using a combination of a nasal septum chondromucosal composite graft and a forehead transposition flap, also known as a "Fricke flap." The flap was designed to include a line of the eyebrow on the lower margin of the flap to replace the eyelash removed during tumor excision. The wound healed completely, without any early or late complications, and the outcome was satisfactory.

Keywords: Eyelid neoplasms / Reconstructive surgical procedures / Sebaceous gland neoplasms

\section{INTRODUCTION}

Sebaceous carcinoma is a malignant neoplasm that usually arises in the sebaceous glands of the eyelids, especially from the meibomian glands of the upper eyelid; the tarsal conjunctiva is the most common site [1,2]. It usually presents as a painless yellow mass with no distinctive clinical features $[2,3]$. The prognosis of sebaceous carcinoma is one of the worst compared to other malignancies occurring in the eyelid, and it accounts for a considerable proportion of malignant eyelid tumors in Asia $[4,5]$.

Wide surgical resection with a safety margin of 5 to $6 \mathrm{~mm}$ is the mainstay of treatment for eyelid sebaceous carcinoma,

\footnotetext{
Correspondence: In Suck Suh

Department of Plastic and Reconstructive Surgery, Kangnam Sacred Heart

Hospital, Hallym University College of Medicine, 1 Singil-ro, Yeongdeungpo-gu,

Seoul 07441, Korea

E-mail: sismdps@gmail.com

Received June 30, 2021 / Revised August 17, 2021 / Accepted August 17, 2021
}

which often results in large full-thickness defects on the eyelid. A three-lamella approach is needed to cover this defect, and a combination of the mucosal component replacing the conjunctiva and the flap component, which has a sufficient blood supply, is ideal.

We present a case of sebaceous carcinoma of the upper eyelid, for which tumor excision and reconstruction using a chondromucosal composite graft and a Fricke flap were performed.

\section{CASE REPORT}

An 88-year-old woman visited our clinic due to a small mass on her right upper eyelid that had developed 6 months prior to her consultation at the department of ophthalmology. The mass was previously proven to be a sebaceous carcinoma by an incisional biopsy done at the department of dermatology. The tumor was located in the lateral region of the right upper eyelid margin, with a size of approximately $5 \mathrm{~mm}$. It was a painless, 
yellow tumor with no other distinctive characteristics (Fig. 1). No regional lymph node involvement or distant metastasis was observed on ultrasonography or magnetic resonance imaging.

As the tumor was on the border of the upper eyelid, we planned a full-thickness wide surgical resection with a safety margin of $5 \mathrm{~mm}$. The full-thickness defect after tumor excision was covered by a combination of a nasal septal chondromucosal graft and a temporal forehead flap.

Surgery was performed under general anesthesia (Fig. 2). The excision of the tumor with a 5-mm safety margin resulted in a 2 -cm-diameter full-thickness defect. The defect was larger than

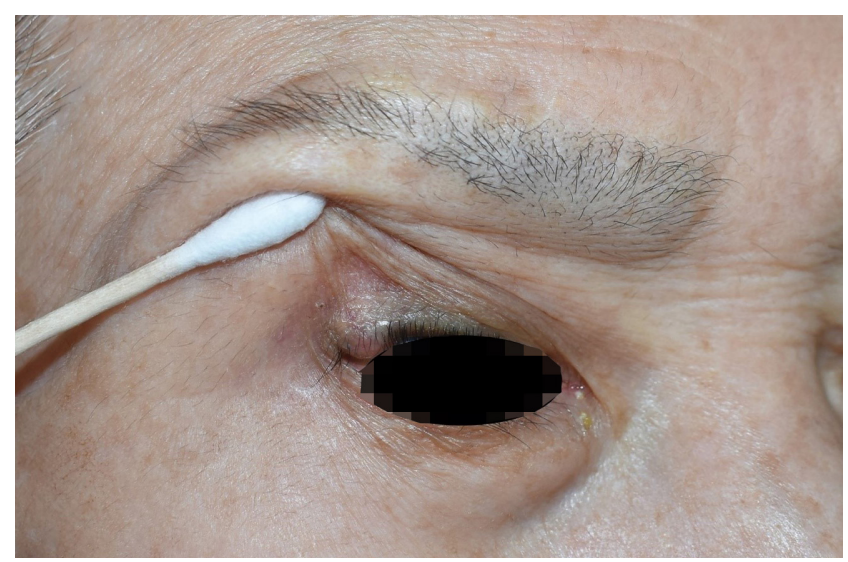

Fig. 1. An 88-year-old woman with a sebaceous carcinoma at the right lateral upper eyelid. one-third of the total length of the upper eyelid. The levator aponeurosis was exposed on the upper margin, the tarsal plate on the medial margin, and the lateral orbital rim on the lateral margin.

Since chondromucosal composite grafts should be harvested with cartilage and mucosa attached, local anesthesia (1:100,000 epinephrine mixed with $2 \%$ lidocaine solution) was administered only between the nasal mucosa and septal cartilage of the opposite side to prevent inappropriate hydrodissection. A $2 \times 3$ $\mathrm{cm}$ graft was then obtained. The septal donor site was covered by a mucosal graft harvested from the buccal mucosa and compressed using a silicone sheet and nasal tampon to prevent septal hematoma. The buccal mucosa donor site was primarily closed.

The harvested graft was trimmed into the shape of the eyelid defect, and the cartilage was sutured with the levator aponeurosis on the upper margin, the orbital rim on the lateral margin, and the tarsal plate on the medial margin.

A Fricke flap, or a forehead transposition flap, was designed just above the eyebrow, including a line of eyebrow that would replace the eyelash excised during wide resection. The width of the flap was set to fit that of the defect, and the length was determined such that the width-to-length ratio did not exceed 1:4 to ensure an appropriate blood supply. The subcutaneous flap was then raised carefully to prevent it from becoming too thick. A tunnel was made between the flap origin and defect, and the
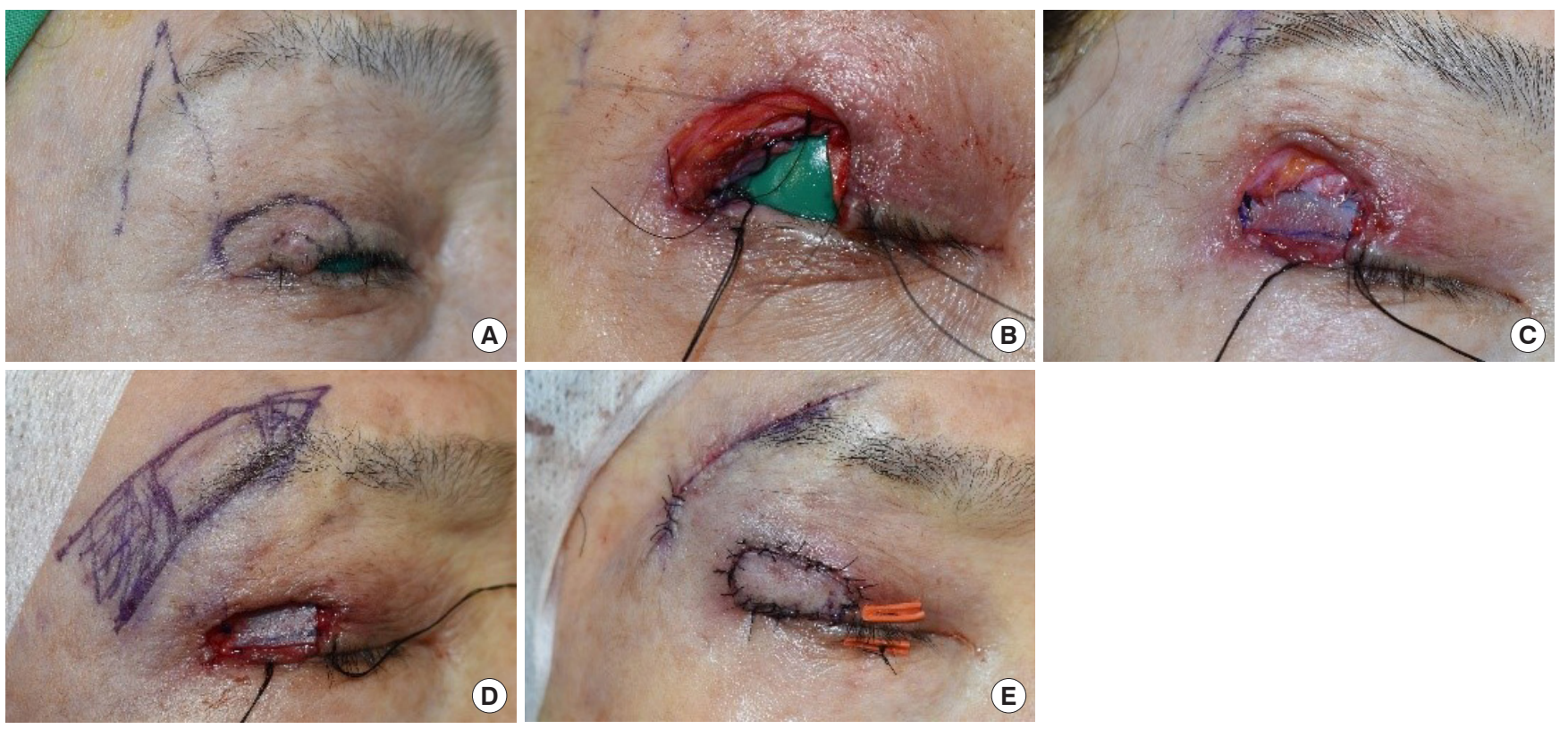

Fig. 2. Intraoperative photographs. (A) Tumor was excised with a safety margin of $5 \mathrm{~mm}$. (B) Full-thickness eyelid defect resulted after tumor excision. (C) Chondromucosal composite graft was harvested from the nasal septum and inserted into the defect. (D) A temporal forehead flap was designed just above the eyebrow including a line of the eyebrow. (E) The flap was passed through the tunnel after de-epithelialization. An appropriately shaped flap was inserted in the proper location. The donor site was closed primarily. 
flap was de-epithelialized, except for the distal portion, to cover the defect. The flap was then passed through the tunnel, and the distal portion of the flap was inserted on the chondromucosal graft. The flap was sutured along the margin of the eyelid defect. The flap donor site was closed primarily, and tarsorrhaphy was performed.

A light compressive dressing and tarsorrhaphy were maintained for a few days, and the stitches were removed after 8 days. Early complications such as hematoma, graft loss, flap necrosis, and nasal septal perforation were not observed.

One year postoperatively, no local recurrence or metastasis was observed on ultrasonography and magnetic resonance imaging, and the wound healed completely without any late complications. Lagophthalmos, scleral show, and asymmetry on both upper eyelids during the opening or closing of the eyes were not observed. The appearance of the eyelashes was satisfactory. There was no trichiasis or alopecia, and the lining of the new eyelash appeared natural in accordance with the adjacent eyelash. The scars of the eyelid and flap donor site were both acceptable. The overall results were satisfactory, and the patient was pleased (Fig. 3).

\section{DISCUSSION}

Eyelid cancers account for $5 \%$ to $10 \%$ of all cutaneous malignancies [6]. Basal cell carcinoma constitutes the majority of all eyelid malignancies, accounting for $86 \%$ to $96 \%$ of all cases, squamous cell carcinoma for $3.4 \%$ to $12.6 \%$, sebaceous carcinoma for $0.6 \%$ to $10.2 \%$, and melanoma and Merkel cell carcinoma for $<1 \%$ each [7]. However, sebaceous carcinoma is more prevalent in Asia, and some studies have reported that sebaceous carcinoma constitutes the majority $(66 \%-67 \%)$ of all eyelid malignancies in Asian populations [5]. Most sebaceous carcinomas occur in patients over 40 years of age, with a median age of 73 years. No difference in prevalence by sex was observed [8].

Wide surgical resection is the mainstay of treatment for seba- ceous carcinoma. The safety margin is $5-6 \mathrm{~mm} \mathrm{[4]} \mathrm{and} \mathrm{if} \mathrm{the}$ tumor invades the orbit, orbital exenteration is indicated [8]. Mohs micrographic surgery and cryotherapy are also treatment options for sebaceous carcinoma, but their indications are limited and their safety is unclear. The efficacy of radiotherapy and chemotherapy remains unknown.

The prognosis of sebaceous carcinoma is one of the worst compared to other malignancies occurring in the eyelid [4]. Sebaceous carcinoma can also metastasize to regional lymph nodes and distant organs. Shields et al. [9] reported recurrence in $18 \%$ and metastasis in $8 \%$ of periocular sebaceous carcinoma cases. The most common sites of distant metastasis are the liver and lung [10], with a 5 -year survival rate of $68 \%$ for periocular sebaceous carcinoma [11]. Computed tomography, magnetic resonance imaging, and positron emission tomography-computed tomography are recommended for detecting regional and distal metastases.

The eyelids are vital structures that protect and lubricate the globes and cornea through a shutter-like mechanism involving the coordination of several components, such as skin, fat, muscle, gland, tarsal plate, eyelash, and conjunctiva. Losing just one of these components can impair the ability of the eyes to blink. Because the eyelids are very small and thin compared to other organs of the face, even small defects could cause the loss of several structures that are difficult to reconstruct.

Tumor resection often results in a full-thickness defect that involves the anterior and posterior lamellae of the eyelid. If the defect is smaller than one-third of the eyelid length, it is not considered to be a complex problem because primary closure with or without cantholysis is possible [12]. However, larger defects of more than one-third of the eyelid length usually appear when malignant tumors are excised. If the defect size is between one-third and half of the upper eyelid, reconstruction using a Tenzel flap, which has a two-step curvature, is the most common technique [6]. Other techniques include local flaps, such as the Mustarde lid-switch pedicle flap and sliding tarsal flap. Since these methods use adjacent tissues, it is easy to replace the
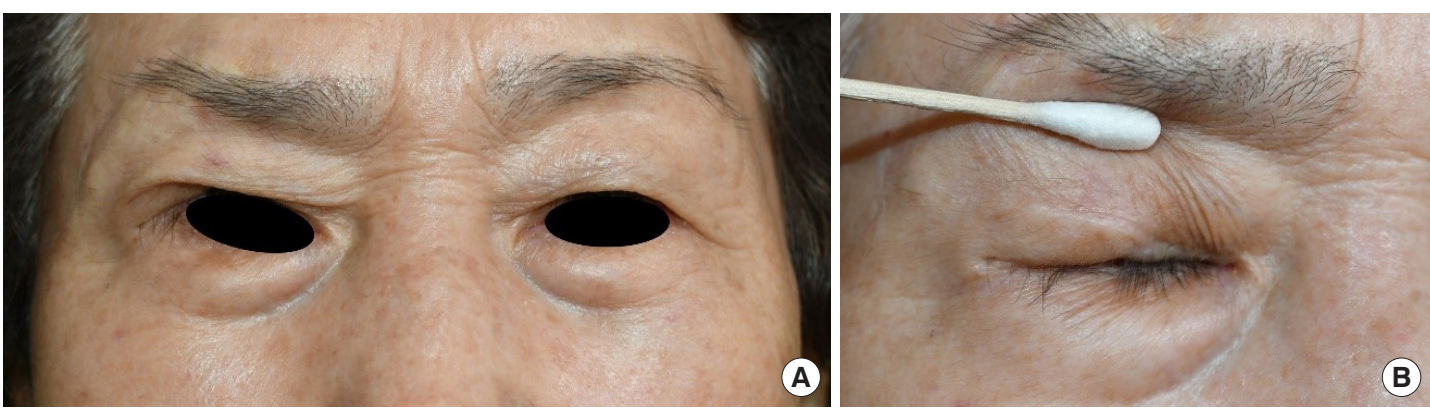

Fig. 3. One-year postoperative photographs. (A, B) No complications were observed, and the appearance was satisfactory. 
excised tissue and avoid donor site morbidity in other areas

In larger defects, which encompass more than half of the eyelid, as in our present case, a three-lamella approach is needed. At least one of the three lamellae (anterior lamella) should consist of a flap for sufficient blood supply; however, the eyelid thickens if two or more lamellae contain flaps [13]. The middle lamella should contain cartilage for the tarsal plate, and the posterior lamella must have a mucosal component to replace the conjunctiva, respectively. Therefore, one local flap and one free graft, including a mucosal component, would be an ideal combination.

The Mustarde lid-switch flap with cheek rotation flap, CutlerBeard lower lid bridge flap, and Fricke flap are commonly used local flaps; in our case, we used the Fricke flap. The Fricke flap, first described by Fricke in 1829, is a temporally based monopedicle forehead transposition flap $[14,15]$. The Fricke flap is commonly used to repair long eyelid defects with a small width and has the advantage of hiding the scar at the upper margin of the eyebrow. We modified the Fricke flap by including a line of the eyebrow in the distal lower margin of the flap to replace the eyelash.

There are several options for graft harvesting, including the mucosal and cartilage components: nasal septal chondromucosal composite graft, ear cartilage, soft palate, and nasal ala. We chose a nasal septal chondromucosal composite graft considering donor site morbidity and recipient suitability because graft harvest on the soft palate results in significant donor site morbidity, the ear cartilage lacks a mucosal component [16], and the nasal alar mucosa is hairy.

The defect caused by harvesting of the mucochondral graft from the nasal septum was repaired with a mucosal graft from the buccal mucosa. Small defects $(<5 \mathrm{~mm})$ in the nasal septum can be healed with conservative treatment or primary closure, but defects larger than $5 \mathrm{~mm}$ result in complications, including septal perforation, if proper surgical treatment is not performed. Septal perforation leads to sequelae such as chondritis, crusting, bleeding, whistling, and rhinorrhea.

The methods of repairing the defect of the nasal septum include a mucosa or skin graft, a local mucosal flap such as interposition or advancement flap, and the use of artificial obturators. Mucosal flaps are good for correcting large defects, but flap procedures often require an open rhinoplasty approach to secure the field of view, take a long time, and have high donor site comorbidity.

Mucosal grafting from the buccal mucosa was performed because it is in the same surgical field as the eyelid and can be primarily closed even for larger defects resulting from mucosal graft harvest. A skin graft wound has also been a treatment op- tion; however, a buccal mucosal graft was chosen because the nature of the skin is very different from that of the mucosa and a visible scar occurs on the skin graft donor site.

After the mucosal graft, a silastic sheet and nasal tampon were applied for a week to prevent hematoma collection in the nasal septum. The mucosal graft was well taken and healed completely without complications such as perforation, chondritis, or hematoma collection.

Full-thickness defects in more than half of the eyelids are uncommon; there are not many options for such defects, and they are difficult to reconstruct. A combination of a Fricke flap and nasal septal chondromucosal composite graft was chosen, and we advanced the technique by adding a line of the eyebrow to repair the eyelash, which is the first line of defense to protect the eyes from foreign bodies (e.g., dust and rain) and makes a major contribution to the aesthetic aspect of the eyes.

\section{NOTES}

\section{Conflict of interest}

No potential conflict of interest relevant to this article was reported.

\section{Ethical approval}

The study was approved by the Institutional Review Board of Kangnam Sacred Heart Hospital (IRB No. 2020-10-004-001) and performed in accordance with the principles of the Declaration of Helsinki.

\section{Patient consent}

The patient provided written informed consent for the publication and the use of her images.

\section{ORCID}

Ju Ho Lee

Sang Seok Woo

Se Ho Shin

Hyeon Jo Kim

Jae Hyun Kim

Seong Hwan Kim

In Suck Suh

https://orcid.org/0000-0002-1958-286X https://orcid.org/0000-0001-6026-4516 https://orcid.org/0000-0002-2761-9327 https://orcid.org/0000-0001-8705-237X https://orcid.org/0000-0002-4041-6284 https://orcid.org/0000-0001-6831-5621 https://orcid.org/0000-0002-9793-4031

\section{Author contribution}

Conceptualization: JHL, SSW, JHK, ISS. Data curation: SSW, HJK. Methodology: ISS. Project administration: SHK. Writing original draft: JHL. Writing - review \& editing: ISS. Investigation: SHS. Software: SHS. Supervision: JHK, ISS. Validation: HJK. 


\section{REFERENCES}

1. Shields JA, Demirci H, Marr BP, Eagle RC Jr, Shields CL. Sebaceous carcinoma of the eyelids: personal experience with 60 cases. Ophthalmology 2004;111:2151-7.

2. Constant E, Leahy MS. Sebaceous cell carcinoma. Plast Reconstr Surg 1968;41:433-7.

3. Lee DW, Kwak SH, Kim JH, Byeon JY, Lee HJ, Choi HJ. Sebaceous carcinoma arising from sebaceoma. Arch Craniofac Surg 2021;22:126-30.

4. Wolfe JT 3rd, Yeatts RP, Wick MR, Campbell RJ, Waller RR. Sebaceous carcinoma of the eyelid: errors in clinical and pathologic diagnosis. Am J Surg Pathol 1984;8:597-606.

5. Chang CH, Chang SM, Lai YH, Huang J, Su MY, Wang HZ, et al. Eyelid tumors in southern Taiwan: a 5-year survey from a medical university. Kaohsiung J Med Sci 2003;19:549-54.

6. Cha JA, Lee KA. Reconstruction of periorbital defects using a modified Tenzel flap. Arch Craniofac Surg 2020;21:35-40.

7. Yin VT, Merritt HA, Sniegowski M, Esmaeli B. Eyelid and ocular surface carcinoma: diagnosis and management. Clin Dermatol 2015;33:159-69.

8. Song X, Jia R, Fan X. An update on eyelid sebaceous cell carcinoma. Int Ophthalmol Clin 2019;59:1-11.

9. Shields JA, Saktanasate J, Lally SE, Carrasco JR, Shields CL. Se- baceous carcinoma of the ocular region: the 2014 Professor Winifred Mao Lecture. Asia Pac J Ophthalmol (Phila) 2015;4: 221-7.

10. Zhou C, Shi Y, Chai P, Wu F, Xia W, He X, et al. Contemporary update of overall prognosis and nomogram to predict individualized survival for Chinese patients with eyelid sebaceous carcinoma. EBioMedicine 2018;36:221-8.

11. Dasgupta T, Wilson LD, Yu JB. A retrospective review of 1349 cases of sebaceous carcinoma. Cancer 2009;115:158-65.

12. Lu GN, Pelton RW, Humphrey CD, Kriet JD. Defect of the eyelids. Facial Plast Surg Clin North Am 2017;25:377-92.

13. Lemaitre S, Levy-Gabriel C, Desjardins L, Gonzalez-Candial M, Gardrat S, Dendale R, et al. Outcomes after surgical resection of lower eyelid tumors and reconstruction using a nasal chondromucosal graft and an upper eyelid myocutaneous flap. J Fr Ophtalmol 2018;41:412-20.

14. Jackson IT. Local flaps in head and neck reconstruction. St. Louis: Mosby; 1985.

15. Fricke JCG. Die Bildung neuer Augenlieder (Blepharoplastik) nach Zerstörungen und dadurch hervorgebrachten Auswärtswendungen derselben. Hamburg: Pethes und Bessler; 1829.

16. Suga H, Ozaki M, Narita K, Kurita M, Shiraishi T, Ohura N, et al. Comparison of nasal septum and ear cartilage as a graft for lower eyelid reconstruction. J Craniofac Surg 2016;27:305-7. 